\title{
IMMUNOGENICITY OF THE MEROZOITE SURFACE PROTEIN-1 (MSP-1) OF HUMAN PLASMODIUM SP.
}

\author{
Cheong FW, Lau YL, Fong MY \\ Faculty of Medicine, University of Malaya, Kuala Lumpur
}

Correspondence:

Associate Prof. Dr. Lau Yee Ling

Department of Parasitology, Faculty of Medicine, University of Malaya, 50603 Kuala Lumpur, Malaysia

Tel: +60379674749

Fax: +60379674754

\begin{abstract}
Malaria is a major cause of mortality and morbidity globally. Great efforts have been made in the prevention and the elimination of malaria, especially in controlling the malaria vector, the mosquito. Another promising approach would be the development of malaria vaccines. Malaria vaccine studies can be focused on the pre-erythrocytic-stage antigens and the blood-stage antigens, and on the transmission blocking agents targeting the malaria gametocytes. The blood-stage antigens are the leading candidates in malaria vaccine development, as the blood-stage parasites are responsible for causing symptomatic malaria. Human acquired immunity largely targets on blood-stage antigens. This review focuses on one of the most extensively studied blood-stage antigen, the merozoite surface protein-1 (MSP-1), specifically on its evaluation and immunogenicity in rodents and primate models, and its safety and immunogenicity in human clinical trials.
\end{abstract}

Keywords: merozoite surface protein, phase trials, Plasmodium, protective immune responses, vaccination

\section{Background}

Malaria remains as one of the important infectious diseases which lead to high global mortality and morbidity annually. According to the World Malaria Report in 2013 (1), there are approximately 207 million clinical cases of malaria each year resulting in 670,000 deaths. About 2.5 billion people, approximately $40 \%$ of the world's population, are at risk. There are five common Plasmodium sp. that naturally infect humans: $P$. falciparum, $P$. vivax, $P$. malariae, $P$. ovale, and $P$. knowlesi. More than $90 \%$ of the global malaria mortality cases occur in sub-Saharan Africa as the majority of the infections in that area is caused by the highly malignant species $P$. falciparum $(2,3)$. Most of the victims are children under 5 years old. Plasmodium vivax has been described as the benign form of malaria, yet many case reports showed that vivax malaria remains responsible for severe clinical manifestations and mortality in vivax-endemic areas (4). Knowlesi-infection is widely distributed in South East Asian countries (5-9) and could lead to hyperparasitemia in a short period as $P$. knowlesi has the fastest life cycle of 24 hours among the human Plasmodium sp. (10). There are limited literature reviews available for $P$. malariae and $P$. Ovale, and this could possibly be due to their low prevalence and milder clinical manifestations compared to the other human Plasmodium sp.
The life cycle of Plasmodium consists of an exoerythrocytic cycle which takes place in the liver, an erythrocytic cycle in the blood circulation and a sporogonic cycle in the mosquito vector. In the erythrocytic cycle, the steps involved are the release of merozoites from the infected hepatocytes, the invasion of merozoites into the erythrocytes, the development and maturation of trophozoites in the erythrocytes, and the release of new merozoites with the rupture of the erythrocytes. This blood-stage life cycle repeats every 24 to 72 hours, with the period dependent on the species of Plasmodium. Clinical illness only occurs in this stage. After repeated exposures, naturally acquired immunity against malaria could develop, predominantly targeting the blood-stage parasites (11). In immunization studies using animal models, a protective immune response is elicited when animals were immunized with blood-stage antigens, particularly merozoite antigens $(12,13)$. Therefore merozoite proteins, either located within the apical organelles or on the merozoite surface, are the leading blood-stage vaccine candidates. To date, the vaccine studies in human trials have been carried out with merozoite surface protein-1 (MSP-1), merozoite surface protein-2, (MSP-2) (14), merozoite surface protein-3 (MSP-3) (15), apical membrane antigen-1 (AMA-1) (16, 17), erythrocyte-binding antigens-175 (EBA-175) (18), glutamate-rich protein (GLURP) (19), serine repeat antigen 
(SERA) (20), circumsporozoite protein (CSP) (21), and sporozoite surface protein (SSP). Among these candidates, merozoite surface protein-1 (MSP-1) is one of the most extensively studied.

MSP-1 is a high molecular mass protein, of 185 to 225 $\mathrm{kDa}$ which fixes at the merozoite surface membrane of the Plasmodium parasite via the glycosyl-phosphatidylinositol (GPI) anchor $(22,23)$. MSP-1 undergoes two steps of processing by proteases and cleaves into a number of fragments. The first processing occurs during the rupture of schizonts, when the merozoites differentiate and are released from an infected erythrocyte. The MSP-1 precursor polypeptide cleaves into four major fragments of different sizes: 83 kDa (MSP-1 ${ }_{83}$ ), 30 kDa (MSP-1 ${ }_{30}$ ), 38 kDa $\left(\mathrm{MSP}-1_{38}\right)$, and $42 \mathrm{kDa}\left(\mathrm{MSP}-1_{42}\right)$ (24). During the invasion of merozoites into the new erythrocytes, the second processing further cleaves MSP-1 $1_{42}$ into two fragments, $33 \mathrm{kDa}\left(\mathrm{MSP}-1_{33}\right.$ ) and $19 \mathrm{kDa}\left(\mathrm{MSP}-1_{19}\right.$ ) (25). The MSP$1_{33^{\prime}}$, corresponding to the $\mathrm{N}$-terminal region of $\mathrm{MSP}-1_{42}$, sheds from the surface in a fully soluble form $(26,27)$, whereas membrane-bound MSP-1 $1_{19}$ remains anchored to the merozoite membrane by the GPI tail attached to the $\mathrm{C}$-terminal residue and will be carried into a new red blood cell $(26,28)$.

Malaria infected individuals who have significantly higher IgG level against N-terminus of MSP-1 are usually asymptomatic compared to the subjects who have acute malaria, demonstrating that anti-MSP-1 antibodies are associated with clinical malarial protection and a reduction of an infection risk $(29,30)$. Studies have also shown that anti-MSP- $1_{42}$, anti-MSP-1 $1_{33}$ and anti-MSP-1 $1_{19}$ could be detected in most of the malaria infected samples, indicating that MSP-1 is one of the immunodominant antigens that could be useful in malaria vaccine development or seroepidemiological screening (31-35). Antibodies directed against MSP-1 $1_{19}$ and MSP-1 $1_{42}$ can interrupt merozoite invasions, and may also inhibit MSP-1 processing (3639). MSP-1 $1_{42}$ and MSP-1 $1_{19}$ are the two fragments that have been most studied, especially in P. falciparum. MSP-1 $1_{19}$ is responsible for humoral immunity responses and a considerable number of studies show that antiMSP- $1_{19}$ antibody plays a role in the protection from symptomatic disease. Children with a naturally acquired immune response to Plasmodium MSP- $1_{19}$ are significantly associated with a resistance towards malaria infection and clinical manifestations (40), while pregnant women with anti-MSP-1 $1_{19}$ antibodies are protected against placental infection and infection in infants (41).

This paper aims to provide an overview on the immunogenicity of MSP-1 and its proteolytic fragments, particularly MSP-1 $1_{42}$ and MSP-1 $1_{19}$. The main issues to be discussed include cell mediated immune response, humoral immune response, protective effects of these fragments in rodent and primate models and human trials. The potential and limitation of MSP-1 as a malaria vaccine candidate will also be discussed.

\section{Immunogenicity of MSP-1 in rodent models}

Before a target antigen could be used in vaccine development, immunization study with animal models is a crucial step to validate the immunogenicity and immunoprotectivity of the target antigen. Rodent models are often chosen as the preliminary animal model due to the ease of handling. In MSP- $1_{42}$ and MSP- $1_{19}$ immunization studies using rodent models, animals were found to be variably protected during a challenge with live Plasmodium parasites. Immunogenicity studies by Dutta et al. (42) in a mice model showed that Escherichia coli (E. coli)-expressed $P$. vivax $\mathrm{MSP}-1_{42}$ induced specific antibody production and lymphocyte proliferation. Protective cytokines, interleukin-2 (IL-2), interleukin-4 (IL-4), interleukin-10 (IL-10) and interferon-gamma (IFN- - ), were detected in the immunized mice. A similar study was carried out on E. coli-expressed P. falciparum MSP-1 ${ }_{42}$, and the rabbitraised anti-MSP-1 ${ }_{42}$ antibodies significantly inhibited the merozoite invasion, while the mice which were passively immunized with anti-MSP-1 ${ }_{42}$ IgG were protected during a challenge with $P$. berghei/P. falciparum chimeric line that expresses $P$. falciparum MSP- $1_{19}$ (43). Cheong et al. (32) also demonstrated that mice immunized with $E$. coli-expressed $P$. knowlesi MSP-1 ${ }_{42}$ exhibited significantly higher levels of IFN- $\psi$, IL-2, IL-4, IL-10 compared to the negative control mice. It is important to take note that MSP- $1_{42}$ exists in nature as two distinct major allelic forms, and immune response produced towards this protein may be greatly affected. Co immunization with $E$. coliexpressed $P$. falciparum and $P$. vivax MSP-1 $1_{19}$ stimulated specific antibody responses against both antigens and the elicited humoral response lasted up to one year after immunization (44). Sachdeva et al. (45) also found that both E. coli-expressed P. vivax MSP- $1_{42}$ and MSP- $1_{19}$ induced specific antibody responses and T-cell responses with six different adjuvants in immunized mice, and high levels of immunoglobulin G1 (IgG1), IL-4, interleukin-5 (IL-5) and IFN- $\gamma$ were detected. Parween et al. (46) showed that the immunogenicity of recombinant $P$. falciparum MSP- $1_{19}$ and $P$. vivax MSP-1 $1_{19}$ was strongly enhanced when the recombinant proteins were coated on Gold Nanoparticles formulated with alum. The raised anti-PfMSP- $1_{19}$ antibodies could inhibit an in vitro merozoite invasion. As opposed to MSP-1 $1_{42}, \mathrm{MSP}_{19}$ is highly conserved but may not provide as much immune response as the longer $\mathrm{MSP}^{-1}{ }_{42}$.

\section{Immunogenicity of MSP-1 in primate models}

Immunization studies in primate models are believed to better resemble the regulation of human immune responses. Numerous immunization studies of MSP-1 42 and $\mathrm{MSP}-1_{19}$ in primate models demonstrated encouraging results, as most of the MSP-1 $1_{42}$ and/or MSP-1 $1_{19}$-immunized non-human primates were significantly protected when challenged with live malaria parasites. For instance, Rogers and co-workers immunized Macaca mulatta with DNA plasmids encoding four $P$. knowlesi antigens including MSP$1_{42}$, and they found that a few of the immunized macaques 
were sterilely protected, while the mean parasitemia in the other macaques was significantly lower than the control macaques during a challenge with $P$. knowlesi sporozoites $(47,48)$. A significantly lower parasitemic level was detected in the rhesus monkeys immunized with $E$. coli-expressed $P$. vivax MSP- $1_{42}$ compared to the negative control group upon a challenge with $P$. cynomolgi, a $P$. vivax-closely related Plasmodium sp., blood stage parasites $(49,50)$. On the other hand, $A$. nancymai vaccinated with $E$. coli-expressed P. falciparum MSP-1 ${ }_{42}$ was highly protected during a lethal $P$. falciparum challenge (51-53), and the protective effect was stronger than the baculovirus-expressed $P$. falciparum MSP-1 ${ }_{42}$ (54). Moreover, specific antibodies and antigenspecific T-cell responses with the production of IFN- $\nu$ were also detected in M. mulatta which were immunized with DNA plasmid encoding $P$. falciparum MSP- $1_{42}$ (55). Before a human trial, pre-clinical analysis on $P$. falciparum MSP-1 formulated with adjuvant ASO2A or alum was performed in $M$. mulatta macaques and both vaccines were shown to be safe and highly immunogenic (56). MSP-1 $1_{42}$ formulated with other adjuvants AS01B, AS05 and ASO8 were also tested in rhesus. All these formulations were found to be safe and immunogenic. AS01B formulation induced a strong Th1 response compared to AS02A which induced a balanced Th1/Th2 response (57). Partial protection was detected in Saimiri boliviensis monkeys upon immunization with yeast-expressed $P$. vivax MSP-1 $19(58,59)$. On the other hand, vaccination of yeast-expressed $P$. falciparum MSP- $1_{19}$ also conferred protection against a lethal challenge of $P$. falciparum in Aotus vociferans monkeys, and the raised anti-MSP- $1_{19}$ antibodies could inhibit the secondary processing of MSP-1 ${ }_{42}(60)$. Efficacy of baculovirusinfected insect cells-expressed $P$. falciparum MSP- ${ }_{42}$ and Saccharomyces cerevisiae (S. cerevisiae)-expressed P. falciparum MSP- $1_{19}$ were compared in an A. nancymai monkey model. A significantly higher protection level was observed in insect cells-expressed $P$. falciparum MSP- $1_{42}{ }^{-}$ vaccinated group compared to $S$. cerevisiae-expressed $P$. falciparum MSP-1 ${ }_{19}$-vaccinated group, and the protection was associated with corresponding antibody levels in the immunized monkeys (61).

\section{MSP-1 in human trials}

Besides animal models, efficacy of MSP-1, including MSP$1_{42}$ and MSP- $1_{19}$ fragments, as a vaccine candidate has also been tested in human trials. Most of the efforts for the development of malaria vaccines and human trials are still focused on $P$. falciparum. Results indicated that MSP-1 formulated with different adjuvants conferred different levels of protection in human clinical trials. Sheehy et al. (62) evaluated the $P$. falciparum MSP-1 in a Phase la clinical trial and induction of exceptionally strong T-cell responses was detected. P. falciparum MSP-1 was tested together with MSP-2 and ring-infected erythrocyte surface antigen (RESA) as a three-component blood-stage vaccine, formulated with Montanide ISA720, in ten male adults (63) and 120 children (64) in a malaria endemic area of Papua New Guinea. The vaccine was found to be safe for use in an already immune population, and MSP1 was shown to be the most immunogenic molecule in that vaccine cocktail, as good cellular response with an increased level of IFN- $\gamma$ and an increase in geometric mean antibody titres against MSP-1 was detected.

The C-terminal MSP- $1_{42}$ has also been tested in human trials besides that of the full length MSP-1. Phase I human vaccine studies by using $P$. falciparum $\mathrm{MSP}-1_{42}$ formulated with adjuvant ASO2A (FMP1/ASO2A) have been carried out in USA with 15 adults. The vaccine was shown to be safe and created minimum reactogenicity with no severe adverse effects in all subjects. A high titre of parasite-reactive antiMSP-1 antibodies was induced and $80 \%$ of immunizedsubject sera reached the minimum functional inhibitory level of $15 \%$ inhibition in parasite growth inhibition assay (65). The same vaccine was evaluated in the falciparummalaria endemic areas, including western Kenya (66) and Mali (67), with 40 adult volunteers in a Phase I trial. The safety and tolerability levels of FMP1/AS02A were high, and the vaccine was highly immunogenic and a statistically significant antibody response was detected. Another Phase I trial was conducted in 135 Kenyan children of ages 12 years to 47 months, and the induced-immune response was dosage-dependent (68). However, a Phase II trial with 400 Kenyan children indicated that FMP1/AS02A may not be a promising candidate for monovalent malaria vaccine, with an overall vaccine efficacy of $5.1 \%$ only (69).

Besides AS02A, MSP-1 ${ }_{42}$ was tested with a few other different adjuvants. The adjuvant Alhydrogel was formulated to Plasmodium falciparum MSP-1 ${ }_{42}$, with both $3 \mathrm{D7}$ and FVO alleles, and evaluated in 60 volunteers in USA. The results showed that although the cytokines IFN- $\gamma$, IL-2, IL-5, IL-10 and IL-13 were detected in the vaccinatedvolunteers, addition of other immunostimulants to both vaccines were needed as the raised anti-MSP-1 $1_{42}$ antibodies were insufficient to inhibit parasite growth up to protection level $(70,71)$. Ellis et al. (72) mixed the FVO and 3D7 of E. coli-expressed $P$. falciparum MSP-1 ${ }_{42}$, formulated with Alhydrogel and novel adjuvant CPG 7909, in order to induce immune responses that recognized the major antigenic polymorphisms. A Phase I trial was carried out in 60 healthy adults. A high safety profile was demonstrated and the encouraging result showed that the sera of MSP$1_{42} /$ CPG 7909-immunized volunteers had an average of $14 \%$, ranging from $3 \%$ to $32 \%$, inhibition activity in the parasite growth inhibition assay. Recently, two Phase I clinical studies on MSP-1 ${ }_{42}$ (FVO) formulated with adjuvant ASO1 were conducted in 26 adults in USA and 30 adults in Kenya (73). The sera of only a few vaccinated-volunteers significantly inhibited parasite growth in vitro. However, the raised-antibodies in USA volunteers exhibited better cross-reactivity to heterologous MSP-1 alleles than the previously tested MSP-1 ${ }_{42}$ (3D7) vaccine.

P. falciparum MSP- $1_{19}$ was combined with domain 3 of apical membrane antigen 1 (AMA1) as a chimeric protein (PfCP-2.9) in human vaccine studies $(74,75)$. This chimeric 
vaccine was formulated with adjuvant Montanide ISA720 and tested in 52 healthy adults (76) and 70 healthy Chinese adults (77), separately. The results demonstrated tolerability and immunogenicity of the formulation, yet optimization evaluations are needed to reduce the reactogenicity. No functional activity against the parasite was observed. Human trials with MSP-1 of other human Plasmodium sp. have not been carried out $(78,79)$.

\section{Conclusion}

Development of malaria vaccines by using MSP-1 encounters numerous challenges. Knowledge about the real functions of this antigen and its interactive mechanisms with human host cells are limited. The approaches of vaccine development to target on functionally important domains/epitopes on MSP-1 are difficult. Human malaria $P$. falciparum and $P$. vivax cannot infect mice. Therefore, vaccine studies of human Plasmodium MSP-1 in non-human models, especially in rodent models, are unable to completely represent the safety, efficacy and immunogenicity of the vaccine targets in human, as there are distinct differences between rodents and human in the immunity regulation and pathogenic responses towards malaria. Most of the non-human primate vaccine studies on MSP-1 alone indicated that only a small percentage of monkeys was protected $(58,59,61)$, either partially or completely, during a challenge of live Plasmodium, while in human vaccine trials, although specific antibodies have been induced, yet the titres were insufficient to neutralize the parasites in vitro. Hence, immunostimulants are needed in order to induce a higher level of protective immune responses. The low level of protection elicited by this single antigen vaccine is an impetus to develop multiantigen vaccines. Nonetheless, the highly immunogenic MSP-1 should remain as one of the potential candidates for blood-stage malaria vaccine design $(32,35,66,67)$. Further investigations and evaluation are needed.

\section{References}

1. World Health Organization. World Malaria Report. Geneva, Switzerland: World Health Organization; 2013.

2. Elliott SR, Beeson JG. Estimating the burden of global mortality in children aged $<5$ years by pathogenspecific causes. Clin Infect Dis. 2008; 46(11):17941795.

3. Snow RW, Guerra CA, Noor AM, Myint HY, Hay SI. The global distribution of clinical episodes of Plasmodium falciparum malaria. Nature. 2005; 434(7030):214217.

4. Anstey NM, Douglas NM, Poespoprodjo JR, Price RN. Plasmodium vivax: clinical spectrum, risk factors and pathogenesis. Adv Parasitol 2012; 80:151-201.
5. Cox-Singh J, Davis TM, Lee KS, et al. Plasmodium knowlesi malaria in humans is widely distributed and potentially life threatening. Clin Infect Dis. 2008; 46(2):165-171.

6. Jiang $\mathrm{N}$, Chang $\mathrm{Q}$, Sun $\mathrm{X}$, et al. Co-infections with Plasmodium knowlesi and other malaria parasites, Myanmar. Emerg Infect Dis. 2010; 16(9):1476-1478.

7. Jongwutiwes S, Buppan P, Kosuvin R, et al. Plasmodium knowlesi Malaria in humans and macaques, Thailand. Emerg Infect Dis. 2011; 17(10):1799-1806.

8. Luchavez J, Espino F, Curameng P, et al. Human Infections with Plasmodium knowlesi, the Philippines. Emerg Infect Dis. 2008; 14(5):811-813.

9. Singh B, Kim Sung L, Matusop A, et al. A large focus of naturally acquired Plasmodium knowlesi infections in human beings. Lancet. 2004; 363(9414):1017-1024.

10. Daneshvar C, Davis TM, Cox-Singh J, et al. Clinical and laboratory features of human Plasmodium knowlesi infection. Clin Infect Dis. 2009; 49(6):852-860.

11. Doolan DL, Dobano C, Baird JK. Acquired immunity to malaria. Clin Microbiol Rev. 2009; 22(1):13-36.

12. Collins $\mathrm{WE}, \mathrm{Pye} \mathrm{D}$, Crewther $\mathrm{PE}$, et al. Protective immunity induced in squirrel monkeys with recombinant apical membrane antigen-1 of Plasmodium fragile. Am J Trop Med Hyg. 1994; 51(6):711-719.

13. Crewther PE, Matthew ML, Flegg RH, Anders RF. Protective immune responses to apical membrane antigen 1 of Plasmodium chabaudi involve recognition of strain-specific epitopes. Infect Immun. 1996; 64(8):3310-3317.

14. McCarthy JS, Marjason J, Elliott S, et al. A phase 1 trial of MSP2-C1, a blood-stage malaria vaccine containing 2 isoforms of MSP2 formulated with Montanide(R) ISA 720. PLoS One. 2011; 6(9):e24413.

15. Audran $\mathrm{R}$, Cachat $\mathrm{M}$, Lurati $\mathrm{F}$, et al. Phase I malaria vaccine trial with a long synthetic peptide derived from the merozoite surface protein 3 antigen. Infect Immun. 2005; 73(12):8017-8026.

16. Malkin EM, Diemert DJ, McArthur JH, et al. Phase 1 clinical trial of apical membrane antigen 1 : an asexual blood-stage vaccine for Plasmodium falciparum malaria. Infect Immun 2005; 73(6): 3677-3685.

17. Saul A, Lawrence $G$, Allworth $A$, et al. A human phase 1 vaccine clinical trial of the Plasmodium falciparum malaria vaccine candidate apical membrane antigen 1 in Montanide ISA720 adjuvant. Vaccine. 2005; 23(23):3076-3083.

18. El Sahly HM, Patel SM, Atmar RL, et al. Safety and immunogenicity of a recombinant nonglycosylated erythrocyte binding antigen 175 Region II malaria vaccine in healthy adults living in an area where malaria is not endemic. Clin Vaccine Immunol. 2010; 17(10):1552-1559.

19. Hermsen CC, Verhage DF, Telgt DS, et al. Glutamaterich protein (GLURP) induces antibodies that inhibit in vitro growth of Plasmodium falciparum in a phase 1 malaria vaccine trial. Vaccine. 2007; 25(15):29302940. 
20. Saul A, Lawrence G, Smillie A, et al. Human phase I vaccine trials of 3 recombinant asexual stage malaria antigens with Montanide ISA720 adjuvant. Vaccine. 1999; 17(23-24):3145-3159.

21. Genton B, Pluschke G, Degen L, et al. A randomized placebo-controlled phase la malaria vaccine trial of two virosome-formulated synthetic peptides in healthy adult volunteers. PLoS One. 2007; 2(10):e1018.

22. Holder AA, Freeman RR. Biosynthesis and processing of a Plasmodium falciparum schizont antigen recognized by immune serum and a monoclonal antibody. J Exp Med. 1982; 156(5):1528-1538.

23. McBride JS, Heidrich HG. Fragments of the polymorphic Mr 185,000 glycoprotein from the surface of isolated Plasmodium falciparum merozoites form an antigenic complex. Mol Biochem Parasitol. 1987; 23(1):71-84.

24. Holder AA, Sandhu JS, Hillman $Y$, et al. Processing of the precursor to the major merozoite surface antigens of Plasmodium falciparum. Parasitology. 1987; 94 (Pt 2):199-208.

25. Blackman MJ, Holder AA. Secondary processing of the Plasmodium falciparum merozoite surface protein-1 (MSP1) by a calcium-dependent membranebound serine protease: shedding of MSP133 as a noncovalently associated complex with other fragments of the MSP1. Mol Biochem Parasitol. 1992; 50(2):307-315.

26. Blackman MJ, Whittle H, Holder AA. Processing of the Plasmodium falciparum major merozoite surface protein-1: identification of a 33-kilodalton secondary processing product which is shed prior to erythrocyte invasion. Mol Biochem Parasitol. 1991; 49(1):35-44.

27. Blackman MJ. Purification of Plasmodium falciparum merozoites for analysis of the processing of merozoite surface protein-1. Methods Cell Biol 1994; 45:213220.

28. Blackman MJ, Ling IT, Nicholls SC, Holder AA. Proteolytic processing of the Plasmodium falciparum merozoite surface protein-1 produces a membranebound fragment containing two epidermal growth factor-like domains. Mol Biochem Parasitol. 1991; 49(1):29-33.

29. Nogueira PA, Alves FP, Fernandez-Becerra C, et al. $A$ reduced risk of infection with Plasmodium vivax and clinical protection against malaria are associated with antibodies against the $\mathrm{N}$ terminus but not the $\mathrm{C}$ terminus of merozoite surface protein 1 . Infect Immun. 2006; 74(5):2726-2733.

30. Versiani FG, Almeida ME, Melo GC, et al. High levels of IgG3 anti ICB2-5 in Plasmodium vivax-infected individuals who did not develop symptoms. Malar J 2013; 12:294.

31. Cheong FW, Lau YL, Fong MY, et al. Evaluation of recombinant Plasmodium knowlesi Merozoite Surface Protein-133 for detection of human malaria. Am J Trop Med Hyg. 2013; 88(5):835-840.

32. Cheong FW, Fong MY, Lau YL, et al. Immunogenicity of bacterial-expressed recombinant Plasmodium knowlesi merozoite surface protein-142 (MSP-142). Malar J. 2013; 12(1):454.

33. Sonaimuthu $\mathrm{P}, \mathrm{Cheong} \mathrm{FW}$, Chin LC, et al. Detection of human malaria using recombinant Plasmodium knowlesi merozoire surface protein-1 (MSP-119) expressed in Escherichia coli. Exp Parasitol 2015; 153:118-122.

34. Lau YL, Cheong FW, Chin LC, et al. Evaluation of codon optimized recombinant Plasmodium knowlesi merozoite surface protein-119 (pkMSP-119) expressed in Pichia pastoris. Trop Biomed. 2014; 31(4):749-759.

35. Versiani FG, Almeida ME, Mariuba LA, et al. N-terminal Plasmodium vivax merozoite surface protein-1, a potential subunit for malaria vivax vaccine. Clin Dev Immunol 2013; 2013:965841.

36. Blackman MJ, Heidrich HG, Donachie S, McBride JS, Holder AA. A single fragment of a malaria merozoite surface protein remains on the parasite during red cell invasion and is the target of invasion-inhibiting antibodies. J Exp Med. 1990; 172(1):379-382.

37. Egan AF, Burghaus $P$, Druilhe $P$, Holder AA, Riley EM. Human antibodies to the $19 \mathrm{kDa}$ C-terminal fragment of Plasmodium falciparum merozoite surface protein 1 inhibit parasite growth in vitro. Parasite Immunol. 1999; 21(3):133-139.

38. O'Donnell RA, de Koning-Ward TF, Burt RA, et al. Antibodies against merozoite surface protein (MSP)-1(19) are a major component of the invasioninhibitory response in individuals immune to malaria. J Exp Med. 2001; 193(12):1403-1412.

39. Blackman MJ, Scott-Finnigan TJ, Shai S, Holder AA. Antibodies inhibit the protease-mediated processing of a malaria merozoite surface protein. J Exp Med. 1994; 180(1):389-393.

40. Riley EM, Allen SJ, Wheeler JG, et al. Naturally acquired cellular and humoral immune responses to the major merozoite surface antigen (PfMSP1) of Plasmodium falciparum are associated with reduced malaria morbidity. Parasite Immunol. 1992; 14(3):321-337.

41. Branch $\mathrm{OH}$, Udhayakumar $\mathrm{V}$, Hightower AW, et al. A longitudinal investigation of IgG and IgM antibody responses to the merozoite surface protein-1 19-kiloDalton domain of Plasmodium falciparum in pregnant women and infants: associations with febrile illness, parasitemia, and anemia. Am J Trop Med Hyg. 1998; 58(2):211-219.

42. Dutta S, Ware LA, Barbosa A, Ockenhouse CF, Lanar DE. Purification, characterization, and immunogenicity of a disulfide cross-linked Plasmodium vivax vaccine candidate antigen, merozoite surface protein 1, expressed in Escherichia coli. Infect Immun. 2001; 69(9):5464-5470.

43. Sachdeva S, Mohmmed A, Dasaradhi PV, et al. Immunogenicity and protective efficacy of Escherichia coli expressed Plasmodium falciparum merozoite surface protein-1(42) using human compatible adjuvants. Vaccine. 2006; 24(12):2007-2016. 
44. Mehrizi AA, Zakeri S, Rafati S, Salmanian AH, Djadid ND. Immune responses elicited by co-immunization of Plasmodium vivax and P. falciparum MSP-1 using prime-boost immunization strategies. Parasite Immunol. 2011; 33(11):594-608.

45. Sachdeva S, Ahmad G, Malhotra P, Mukherjee P, Chauhan VS. Comparison of immunogenicities of recombinant Plasmodium vivax merozoite surface protein 1 19- and 42-kiloDalton fragments expressed in Escherichia coli. Infect Immun. 2004; 72(10):57755782.

46. Parween S, Gupta PK, Chauhan VS: Induction of humoral immune response against PfMSP-1(19) and PVMSP-1(19) using gold nanoparticles along with alum. Vaccine 2011; 29:2451-2460.

47. Rogers WO, Weiss WR, Kumar A, et al. Protection of rhesus macaques against lethal Plasmodium knowlesi malaria by a heterologous DNA priming and poxvirus boosting immunization regimen. Infect Immun. 2002; 70(8):4329-4335.

48. Rogers WO, Baird JK, Kumar A, et al. Multistage multiantigen heterologous prime boost vaccine for Plasmodium knowlesi malaria provides partial protection in rhesus macaques. Infect Immun. 2001; 69(9):5565-5572.

49. Dutta S, Kaushal DC, Ware LA, et al. Merozoite surface protein 1 of Plasmodium vivax induces a protective response against Plasmodium cynomolgi challenge in rhesus monkeys. Infect Immun. 2005; 73(9):59365944.

50. Kaushal DC, Kaushal NA, Narula A, et al. Biochemical and immunological characterization of $E$. coli expressed $42 \mathrm{kDa}$ fragment of Plasmodium vivax and $P$. cynomolgi bastianelli merozoite surface protein-1. Indian J Biochem Biophys. 2007; 44(6):429-436.

51. Singh $S$, Kennedy $M C$, Long $C A$, Saul AJ, Miller LH, Stowers AW. Biochemical and immunological characterization of bacterially expressed and refolded Plasmodium falciparum 42-kilodalton C-terminal merozoite surface protein 1. Infect Immun. 2003; 71(12):6766-6774.

52. Singh S, Miura K, Zhou H, et al. Immunity to recombinant Plasmodium falciparum merozoite surface protein 1 (MSP1): protection in Aotus nancymai monkeys strongly correlates with antiMSP1 antibody titer and in vitro parasite-inhibitory activity. Infect Immun. 2006; 74(8):4573-4580.

53. Darko CA, Angov E, Collins WE, et al. The clinical-grade 42-kilodalton fragment of merozoite surface protein 1 of Plasmodium falciparum strain FVO expressed in Escherichia coli protects Aotus nancymai against challenge with homologous erythrocytic-stage parasites. Infect Immun. 2005; 73(1):287-297.

54. Lyon JA, Angov E, Fay MP, et al. Protection induced by Plasmodium falciparum MSP1(42) is strain-specific, antigen and adjuvant dependent, and correlates with antibody responses. PLoS One. 2008; 3(7):e2830.

55. Kumar $\mathrm{S}$, Villinger $\mathrm{F}$, Oakley $\mathrm{M}$, et al. A DNA vaccine encoding the $42 \mathrm{kDa}$ C-terminus of merozoite surface protein 1 of Plasmodium falciparum induces antibody, interferon-gamma and cytotoxic $T$ cell responses in rhesus monkeys: immuno-stimulatory effects of granulocyte macrophage-colony stimulating factor. Immunol Lett. 2002; 81(1):13-24.

56. Angov E, Aufiero BM, Turgeon AM, et al. Development and pre-clinical analysis of a Plasmodium falciparum Merozoite Surface Protein-1(42) malaria vaccine. Mol Biochem Parasitol. 2003; 128(2):195-204.

57. Pichyangkul S, Gettayacamin M, Miller RS, et al. Pre-clinical evaluation of the malaria vaccine candidate $P$. falciparum MSP1(42) formulated with novel adjuvants or with alum. Vaccine. 2004; 22(2930):3831-3840.

58. Collins WE, Kaslow DC, Sullivan JS, et al. Testing the efficacy of a recombinant merozoite surface protein (MSP-1(19) of Plasmodium vivax in Saimiri boliviensis monkeys. Am J Trop Med Hyg. 1999; 60(3):350-356.

59. Yang C, Collins WE, Sullivan JS, Kaslow DC, Xiao L, Lal AA. Partial protection against Plasmodium vivax blood-stage infection in Saimiri monkeys by immunization with a recombinant C-terminal fragment of merozoite surface protein 1 in block copolymer adjuvant. Infect Immun. 1999; 67(1):342349.

60. Egan AF, Blackman MJ, Kaslow DC. Vaccine efficacy of recombinant Plasmodium falciparum merozoite surface protein 1 in malaria-naive, -exposed, and/ or -rechallenged Aotus vociferans monkeys. Infect Immun. 2000; 68(3):1418-1427.

61. Stowers AW, Cioce V, Shimp RL, et al. Efficacy of two alternate vaccines based on Plasmodium falciparum merozoite surface protein 1 in an Aotus challenge trial. Infect Immun. 2001; 69(3):1536-1546.

62. Sheehy SH, Duncan CJ, Elias SC, et al. Phase la clinical evaluation of the Plasmodium falciparum blood-stage antigen MSP1 in ChAd63 and MVA vaccine vectors. Mol Ther. 2011; 19(12):2269-2276.

63. Genton B, Al-Yaman F, Anders R, et al. Safety and immunogenicity of a three-component blood-stage malaria vaccine in adults living in an endemic area of Papua New Guinea. Vaccine. 2000; 18(23):2504-2511.

64. Genton B, Al-Yaman F, Betuela I, et al. Safety and immunogenicity of a three-component bloodstage malaria vaccine (MSP1, MSP2, RESA) against Plasmodium falciparum in Papua New Guinean children. Vaccine. 2003; 22(1):30-41.

65. Ockenhouse CF, Angov E, Kester KE, et al. Phase I safety and immunogenicity trial of FMP1/AS02A, a Plasmodium falciparum MSP-1 asexual blood stage vaccine. Vaccine. 2006; 24(15):3009-3017.

66. Stoute JA, Gombe J, Withers MR, et al. Phase 1 randomized double-blind safety and immunogenicity trial of Plasmodium falciparum malaria merozoite surface protein FMP1 vaccine, adjuvanted with AS02A, in adults in western Kenya. Vaccine. 2007; 25(1):176-184.

67. Thera MA, Doumbo OK, Coulibaly D, et al. Safety and allele-specific immunogenicity of a malaria vaccine in 
Malian adults: results of a phase I randomized trial. PLoS Clin Trials. 2006; 1(7):e34.

68. Withers MR, McKinney D, Ogutu BR, et al. Safety and reactogenicity of an MSP-1 malaria vaccine candidate: a randomized phase Ib dose-escalation trial in Kenyan children. PLoS Clin Trials. 2006; 1(7):e32.

69. Ogutu BR, Apollo OJ, McKinney D, et al. Blood stage malaria vaccine eliciting high antigen-specific antibody concentrations confers no protection to young children in Western Kenya. PLoS One. 2009; 4(3):e4708.

70. Malkin E, Long CA, Stowers AW, et al. Phase 1 study of two merozoite surface protein 1 (MSP1(42)) vaccines for Plasmodium falciparum malaria. PLoS Clin Trials. 2007; 2(4):e12.

71. Huaman MC, Martin LB, Malkin E, et al. Ex vivo cytokine and memory $T$ cell responses to the 42kDa fragment of Plasmodium falciparum merozoite surface protein-1 in vaccinated volunteers. J Immunol. 2008; 180(3):1451-1461.

72. Ellis RD, Martin LB, Shaffer D, et al. Phase 1 trial of the Plasmodium falciparum blood stage vaccine MSP1(42)-C1/Alhydrogel with and without CPG 7909 in malaria naive adults. PLoS One. 2010; 5(1):e8787.

73. Otsyula N, Angov E, Bergmann-Leitner E, et al. Results from tandem Phase 1 studies evaluating the safety, reactogenicity and immunogenicity of the vaccine candidate antigen Plasmodium falciparum FVO merozoite surface protein-1 (MSP1(42)) administered intramuscularly with adjuvant system AS01. Malar J. 2013; 12(1):29.

74. Pan W, Huang $D$, Zhang $\mathrm{Q}$, et al. Fusion of two malaria vaccine candidate antigens enhances product yield, immunogenicity, and antibody-mediated inhibition of parasite growth in vitro. J Immunol. 2004; 172(10):6167-6174.

75. Langermans JA, Hensmann $M$, van Gijlswiik $M$, et al. Preclinical evaluation of a chimeric malaria vaccine candidate in Montanide ISA 720: immunogenicity and safety in rhesus macaques. Hum Vaccin. 2006; 2(5):222-226.

76. Hu J, Chen Z, Gu J, et al. Safety and immunogenicity of a malaria vaccine, Plasmodium falciparum AMA-1/ MSP-1 chimeric protein formulated in montanide ISA 720 in healthy adults. PLoS One. 2008; 3(4):e1952.

77. Malkin E, Hu J, Li Z, et al. A phase 1 trial of PfCP2.9: an AMA1/MSP1 chimeric recombinant protein vaccine for Plasmodium falciparum malaria. Vaccine. 2008; 26(52):6864-6873.

78. Arevalo-Herrera M, Chitnis C, Herrera S. Current status of Plasmodium vivax vaccine. Hum Vaccin. 2010; 6(1):124-132.

79. Richards JS, Beeson JG. The future for blood-stage vaccines against malaria. Immunol Cell Biol. 2009; 87(5):377-390. 